\title{
Conocimiento e investigación sobre la autonomía profesional en enfermería. Revisión narrativa
}

\author{
Knowledge and research about nursing \\ professional autonomy. Narrative review
}

\section{Conhecimento e pesquisa sobre autonomia profissional em enfermagem. Revisão narrativa}

\author{
A. Hine-Sanabriaa , A. Mendoza-Monsalvea, L.Z. Rojas' ${ }^{b}$, Z.M. Roa-Díaz ${ }^{c^{*}}$ \\ aEscuela de Enfermería, Facultad de Salud, Universidad Industrial de Santander, Bucaramanga, Santander, \\ Colombia \\ ${ }^{\text {b} G r u p o ~ d e ~ I n v e s t i g a c i o ́ n ~ y ~ D e s a r r o l l o ~ d e ~ C o n o c i m i e n t o ~ e n ~ E n f e r m e r i ́ a ~ F C V ~(G I D C E N-F C V), ~ C e n t r o ~ d e ~ I n v e s t i-~}$ \\ gaciones, Fundación Cardiovascular de Colombia, Bucaramanga, Santander, Colombia \\ `Grupo de Investigación en Enfermería (GRINFER) Escuela de Enfermería, Facultad de Salud, Universidad In- \\ dustrial del Santander. Bucaramanga, Santander, Colombia
}

Recibido: 7 marzo 2017

Aceptado: 9 febrero 2018

\section{Resumen}

Introducción: Enfermería como disciplina se encuentra en un momento trascendental a la luz de los desarrollos científicos que están en espera de ser incorporados en el cuidado y que necesitan enfermeros autónomos en su práctica profesional, por lo cual en el presente trabajo se realizó una revisión narrativa del concepto de autonomía.

Objetivo: Describir el concepto, medición e investigaciones sobre la autonomía profesional en estudiantes de enfermería.

Desarrollo: La autonomía profesional ha acompañado la evolución de la enfermería; ha pasado por momentos de invisibilidad hasta ser parte de la definición de la profesión y su práctica. Por lo anterior, la medición de este constructo ha cobrado gran importancia, identificándose tres categorías, en primer lugar se encuentran los instrumentos que buscan explorar la actitud de la enfermera y las características de los pacientes, en segundo lugar, los que fijan su perspectiva desde el desempeño laboral de la enfermera y por último se destacan aquellos que hacen una diferenciación de la medición según características como sexo y experticia; estos instrumentos han sido empleados en estudios principalmente transversales que han establecido comparaciones con otras profesiones.

*Autor para correspondencia, email: zaynemilena6@gmail.com. (Z.M. Roa Díaz) 
Conclusiones: Dada la importancia del concepto de autonomía y su escasa descripción, medición y evaluación como constructo de la identidad profesional en enfermería, así como la escasez de trabajos publicados en español; se identificó la necesidad de realizar investigaciones con instrumentos válidos, en diseños longitudinales que establezcan los factores asociados al desarrollo de la autonomía profesional y contribuyan al fortalecimiento de esta característica en el personal de enfermería.

Palabras clave: Autonomía profesional; estudiantes de enfermería; investigación en enfermería; psicometría; Colombia.

\begin{abstract}
Introduction: The discipline of Nursing is situated in a transcendental moment because, among other, the scientific developments which are being incorporated into the healthcare process are to be utilized by autonomous nurses. Thus, this study is aimed at narratively reviewing the concept of autonomy.

Objective:To describe the concept, measurement, and related research, regarding professional autonomy among nursing students.

Development: Professional autonomy has accompanied the evolution of nursing from, at some points being invisible, to now being an explicit part of the profession's definition and practice. Because of this, the construct's measurement has become more important. Three types of instruments have been suggested: those with a focus in the exploration of attitudes of nurses and patients; those with a perspective towards the nurses' performance; and those based on specific characteristics such as sex and expertise. These instruments have been mainly used in transversal studies for comparisons.

Conclusions: Given the importance of the concept, considering the scant information related to the description, measurement, and assessment as a nursing professional identity construct, and also considering the scant Spanish-published related studies, a need was identified to do research with longitudinal designs and validated instruments which can reveal the factors associated with the development and strengthening of professional autonomy in nursing personnel.
\end{abstract}

Keywords: Professional autonomy; students, nursing; nursing research; psychometry; Colombia.

\title{
Resumo
}

Introdução: A enfermagem como disciplina encontra-se em um momento transcendental à luz dos desenvolvimentos científicos que estão na espera de ser incorporados no cuidado, e que necessitam enfermeiros autônomos em sua prática profissional, pelo qual no presente trabalho realizou-se uma revisão narrativa do conceito de autonomia.

Objetivo: Descobrir o conceito, medição e pesquisas sobre a autonomia profissional em estudantes de enfermagem.

Desenvolvimento: A autonomia profissional tem acompanhado a evolução da enfermagem; tem passado por momentos de invisibilidade até ser parte da definição da profissão e sua prática. Portanto, a medição deste construto cobrou grande importância, identificando-se três categorias, em primeiro lugar encontram-se os instrumentos que buscam explorar a atitude da enfermeira e as características dos pacientes, em segundo lugar, os que fixam sua perspectiva desde o desempenho laboral da enfermeira e por último destacam-se aqueles que fazem uma diferenciação da medição segundo características como sexo e expertise; estes instrumentos têm sido empregados em estudos principalmente transversais que estabeleceram comparações com outras profissões.

Conclusões: Dada a importância do conceito de autonomia e sua escassa descrição, medição e avaliação como constructo da identidade profissional em enfermagem, assim como a escassez de trabalhos publicados em espanhol; identificou-se a necessidade de realizar pesquisas com instrumentos válidos, 
em desenhos longitudinais que estabeleçam os fatores associados ao desenvolvimento da autonomia profissional e contribuam no reforço desta característica no pessoal de enfermagem.

Palavras chave: Autonomia profissional; estudantes de enfermagem; pesquisa em enfermagem; psicometria; Colômbia.

\section{Introducción}

El Consejo Internacional de Enfermería define la profesión como aquella que [...] abarca los cuidados autónomos y en colaboración, que se prestan a las personas $[\ldots]^{1}$, igualmente países como Colombia a través de la ley 911 de 2004 declara la autonomía profesional como una condición para el ejercicio de la enfermería ${ }^{2}$, estos planteamientos son coherentes con la posición de algunos autores que consideran la autonomía profesional como un factor clave en la práctica de la enfermería basada en la evidencia $(\mathrm{EBE})^{3}$.

En este sentido la autonomía es un concepto central en el control del comportamiento humano de amplio interés desde la perspectiva profesional como una característica que influye en la toma de decisiones y juicios acerca de los servicios que se prestan, contribuye al manejo de presiones externas, incluidas las generadas por empleadores, legisladores del gobierno, reguladores e incluso otros profesionales 5 .

Así pues, el constructo autonomía ha sido definido como la habilidad para desempeñar funciones en forma independiente sin tener supervisión cercana ${ }^{6}$, dicha conceptualización reitera su importancia en la demostración del estatus de profesión, al aplicar un único cuerpo de conocimiento disciplinar ${ }^{7}$; asimismo, el fortalecimiento de la autonomía es esencial para reducir la probabilidad de aparición de consecuencias negativas como sufrimiento y muerte, tanto en los sujetos de cuidado, como la aparición de estados de insatisfacción profesional o agotamiento en los enfermeros ${ }^{8}$. Dada la relevancia del constructo autonomía para la enfermería, se realizó el presente trabajo a partir de la literatura disponible, el objetivo es describir el concepto y medición, así como investigaciones sobre la autonomía profesional en estudiantes de enfermería.

\section{Métodos}

Esta revisión narrativa de la literatura, se fundamentó en una estrategia de búsqueda con los términos MeSH "Professional Autonomy" AND "Students, nursing” en las bases de datos de Pubmed y Web of Science. Con el propósito de dar respuesta a las siguientes preguntas: ¿Cuál es el concepto de la autonomía profesional de enfermería?; ¿Cuáles instrumentos existen para la medición la autonomía profesional en enfermería?; ¿Qué investigaciones sobre o alrededor de la autonomía profesional se han desarrollado en estudiantes de enfermería?

\section{Desarrollo}

Definición y evolución del concepto de autonomía, desde la perspectiva de enfermería.

El término autonomía, tal como se entiende hoy, viene del griego autos (sí mismo) y nomos (ley). Por lo tanto, hace referencia a la posibilidad que tiene todo ser humano de adoptar sus propias normas para la realización de su vida, sin esperar premios ni castigos por las acciones que ejecuta, sino tan solo por la satisfacción que conlleva la propia realización?

La autonomía como principio no es una condición inherente a todos los seres humanos, puede decirse que se es autónomo en la medida en que el uso de la libertad es coherente con la responsabilidad que se tiene consigo mismo, con los demás y con la sociedad. De tal forma, la autonomía es algo que se alcanza con el paso del tiempo y se es autónomo, en la medida en que se es capaz de dictar las propias normas, en que no se necesita de instancias reguladoras o sancionatorias para hacer las cosas; 
la autonomía va íntimamente ligada con el uso adecuado de la libertad y con el sentido de la responsabilidad que se tiene frente a las cosas?.

Por otra parte, la autonomía profesional ha acompañado la evolución de la enfermería; se identifican momentos en que su presencia estuvo implícita en la práctica sin definición evidente, hasta debates teóricos que tras algún tiempo han logrado llevar el concepto a la definición misma del quehacer de la profesión; a continuación se presentan momentos claves en el desarrollo de la profesión de enfermería y el constructo de autonomía.

A inicios del siglo XVIII, antes de la industrialización, la enfermería funcionó de forma independiente en virtud del contexto de la práctica y la simplicidad de la asistencia sanitaria ${ }^{10}$. En la década de 1930, con la aparición de los hospitales surgió un nuevo contexto para el ejercicio de la profesión, caracterizado por elementos como la burocracia y el patriarcado que alteraron la independencia y percepción de la autonomía en el personal de enfermería, situación que promovió la identificación conceptual y práctica de este constructo ${ }^{8}$.

En 1965 Ghristman, como lo mencionan Pankarts et al. ${ }^{10}$ identificó que la enfermería era una carrera seleccionada, predominantemente, por mujeres que luego eran dominadas por los profesionales varones. Lo anterior estableció las bases de una corriente de pensamiento que diferenció la autonomía de acuerdo al sexo; se argumenta que las mujeres desarrollan la autonomía en el contexto de las relaciones y la ética del cuidado, mientras que la autonomía en los hombres se asocia con la orientación al logro y la agresividad interpersonal ${ }^{11}$.

Tras algunas décadas de transición entre los desarrollos conceptuales y contextos para el cuidado, se formularon definiciones independientes de otras profesiones, como lo presenta Pankarts et al. ${ }^{10}$ al señalar que Kinlein, presentó la práctica de enfermería como una ciencia completamente separada de cualquier médico u otro profesional de salud; señaló que la enfermería se ve como una extensión de la persona al cuidado, más no del médico. Más adelante, esta corriente de pensamiento fue favorecida por los enfoques de atención que daban importancia a la creación de relaciones de colaboración interprofesional médico-enfermera, para ayudar a controlar los costos de cuidado de la salud y la toma de decisiones satisfactorias ${ }^{12}$, lo que marcó un reconocimiento social y científico como disciplina independiente.

Finalmente, la enfermería a principios del Siglo XX, pasa de ser un oficio a ser legalmente reconocida como profesión ${ }^{13}$, éste hecho contribuyó a fortalecer la autonomía profesional; desde entonces, los profesionales de enfermería y otros coinciden en que la situación profesional no puede alcanzarse sin autonomía ${ }^{11}$; sin embargo, aún no se tiene una definición clara y ampliamente aceptada de autonomía profesional en enfermería, lo cual, como se presenta a continuación, ha generado la creación de instrumentos con diferentes enfoques.

\section{Instrumentos para medir autonomía en la profesión de enfermería}

Existen diferencias en los instrumentos diseñados para medir autonomía desde la perspectiva de la profesión de enfermería, esta diversidad es consecuencia del desarrollo conceptual de este constructo. Por lo anterior, y con el fin de facilitar la revisión de las propiedades psicométricas de dichos instrumentos, en el presente trabajo se han clasificado teniendo en cuenta su enfoque de desarrollo, diferenciándose tres categorías, en primer lugar se encuentran los instrumentos que buscan explorar la actitud de la enfermera y características de los pacientes, en segundo lugar, los que fijan su perspectiva desde el desempeño laboral de la enfermera ${ }^{14}$ y por último, se destacan aquellos que hacen una diferenciación de la medición según características como sexo y experticia ${ }^{15}{ }^{16}$.

Dentro de la primera categoría, en la cual se identifican los instrumentos que involucran características de los sujetos de cuidado, se encuentra el Nurse Autonomy and Patient Rights Scale (NAPRS) ${ }^{10,17}$, este instrumento ampliamente utilizado, cuenta con trabajos que reportan sus hallazgos o la evaluación de sus propiedades psicométricas en diferentes poblaciones, entre ellas, estudiantes de enfermería y 
enfermeros con o sin formación de posgrado de varios países. De sus propiedades psicométricas es importante destacar los altos coeficientes que se han obtenido en diferentes procesos de validación; lo cuales son un importante respaldo de su confiabilidad (Tabla 1); sin embargo, se encuentra heterogeneidad en el reporte de dichas propiedades, puesto que algunos estudios presentan información sólo para sus subescalas ${ }^{18,17}$, lo cual impide una evaluación y comparación global. Por otra parte, este instrumento es fuertemente criticado al considerarse que su contenido representa el constructo -independencia- el cuál ha tenido un amplio debate y definición en la literatura y ha sido claramente diferenciado del constructo de autonomía ${ }^{14}$.

Tabla 1. Instrumentos para medir autonomía: concepto medido, confiabilidad y validez

\begin{tabular}{|c|c|c|c|c|c|}
\hline Autor (año) & Instrumento & $\begin{array}{l}\text { Concepto } \\
\text { medido }\end{array}$ & Definición Concepto & Confiabilidad & Validez \\
\hline $\begin{array}{l}\text { Pankratz \& } \\
\text { Pankratz }(1974)^{10}\end{array}$ & $\begin{array}{l}\text { Nurse Autonomy } \\
\text { and Patient } \\
\text { Rights Scale }\end{array}$ & $\begin{array}{l}\text { Autonomía } \\
\text { de } \\
\text { enfermeras } \\
\text { y pacientes }\end{array}$ & $\begin{array}{l}\text { Funcionamiento } \\
\text { independiente en } \\
\text { ámbito de ejercicio } \\
\text { profesional individual }\end{array}$ & $\alpha=0.81-0.93$ & $\begin{array}{l}\text { Análisis de } \\
\text { factores, contraste } \\
\text { de grupos }\end{array}$ \\
\hline $\begin{array}{l}\text { Schutzenhofer } \\
(1988)^{19}\end{array}$ & $\begin{array}{l}\text { Nursing } \\
\text { Activity Scale }\end{array}$ & $\begin{array}{l}\text { Autonomía } \\
\text { clínica }\end{array}$ & $\begin{array}{l}\text { Práctica profesional } \\
\text { acorde con la educación } \\
\text { y leyes laborales, que } \\
\text { definan y controlen sus } \\
\text { actividades en ausencia } \\
\text { de controles externos. }\end{array}$ & $\begin{array}{l}\alpha=0.86 \text {; test- } \\
\text { retest } r=0.88\end{array}$ & $\begin{array}{l}\text { Panel expertos, } \\
\text { contraste de } \\
\text { grupos }\end{array}$ \\
\hline $\begin{array}{l}\text { Papathanassoglou, } \\
\text { et al. }(2005)^{20}\end{array}$ & $\begin{array}{l}\text { Hellenic } \\
\text { Intensive } \\
\text { Care Nurses } \\
\text { Autonomy Scale }\end{array}$ & $\begin{array}{l}\text { Autonomía } \\
\text { clínica }\end{array}$ & $\begin{array}{l}\text { Creencia en la persona } \\
\text { como eje central al } \\
\text { momento de tomar } \\
\text { decisiones responsables, } \\
\text { independientes e } \\
\text { interdependientes. }\end{array}$ & $\begin{array}{l}\alpha=0.86 \text {; test- } \\
\text { retest } r=0.88\end{array}$ & $\begin{array}{l}\text { Análisis factorial, } \\
\text { contraste de } \\
\text { grupos }\end{array}$ \\
\hline De Jonge $(1995)^{21}$ & $\begin{array}{l}\text { Maastricht } \\
\text { Autonomy Scale }\end{array}$ & $\begin{array}{l}\text { Autonomía } \\
\text { en el Trabajo }\end{array}$ & $\begin{array}{l}\text { Libertad en el trabajo para } \\
\text { determinar los elementos } \\
\text { de la tarea, incluidos el } \\
\text { método, orden, cantidad } \\
\text { y la evaluación, }\end{array}$ & $\begin{array}{l}a=0.81- \\
0.88 ; \text { test- } \\
\text { retest } 8 \text { meses } \\
r=0.66\end{array}$ & $\begin{array}{l}\text { Panel expertos, } \\
\text { análisis factorial, } \\
\text { Evaluación de } \\
\text { ajuste del modelo } \\
\text { y validez predictiva }\end{array}$ \\
\hline $\begin{array}{l}\text { Dwyer \& Ganster } \\
(1991)^{22}\end{array}$ & Control Scale & $\begin{array}{l}\text { Autonomía } \\
\text { en el Trabajo }\end{array}$ & $\begin{array}{l}\text { Margen de decisión en } \\
\text { el ambiente de trabajo. }\end{array}$ & $\alpha=0.87$ & Análisis factorial \\
\hline $\begin{array}{l}\text { Haynes, Wall, } \\
\text { Bolden, Stride, \& } \\
\text { Rick }(1999)^{23}\end{array}$ & $\begin{array}{l}\text { Autonomy and } \\
\text { Control Scale }\end{array}$ & $\begin{array}{l}\text { Autonomía } \\
\text { en el trabajo }\end{array}$ & $\begin{array}{l}\text { Alcance de las personas } \\
\text { para elegir como llevar } \\
\text { a cabo su trabajo. }\end{array}$ & $\alpha=0.88$ & $\begin{array}{l}\text { Análisis factorial, } \\
\text { evaluación de } \\
\text { ajuste del modelo } \\
\text { y contraste } \\
\text { de grupos. }\end{array}$ \\
\hline Boughn $(1995)^{16}$ & $\begin{array}{l}\text { Autonomy: } \\
\text { The Caring } \\
\text { Perspective }\end{array}$ & $\begin{array}{l}\text { Las actitudes } \\
\text { hacia la } \\
\text { autonomía } \\
\text { del paciente }\end{array}$ & $\begin{array}{l}\text { Capacidad de cuidado y } \\
\text { defensa de los demás. }\end{array}$ & $\begin{array}{l}\alpha=0.84 \\
\text { test- retest } \\
2 \text { semanas } \\
r=0.90\end{array}$ & $\begin{array}{l}\text { IVC }=0.77 \text {, análisis } \\
\text { de factores, } \\
\text { contraste de } \\
\text { grupos y validez } \\
\text { convergente. }\end{array}$ \\
\hline
\end{tabular}

En segundo lugar, se encuentran las escalas centradas en el desempeño laboral de la enfermera, las cuales han sido evaluadas principalmente en profesionales graduados, debido a que involucran condiciones externas para el ejercicio de la profesión. En esta categoría es importante destacar que se identifican dos conceptos sutilmente diferentes, que a su vez agrupan instrumentos, el primero es la autonomía en el trabajo y en segundo lugar se encuentra la autonomía clínica ${ }^{14}$, de acuerdo a lo 
anterior, la autonomía clínica se define como "la aplicación de conocimientos y habilidades de enfermería en el cuidado ejecutado en una práctica interdependiente, que incluso llega a requerir acciones por parte de la enfermera que van más allá de la norma habitual" ${ }^{14}$, en este concepto se pueden distinguir, principalmente, tres instrumentos el Nursing Activity Scale ${ }^{19}$, Hellenic Intensive Care Nurses Autonomy Scale ${ }^{20}$, para las cuales se han reportado Alfas de Cronbach superiores a 0.80 (Tabla 1), también es importante mencionar que la escala Hellenic Intensive Care cuenta con evidencia que la respalda como la más reproducible de las tres anteriormente mencionadas, así como su mayor capacidad de explicar la varianza total del constructo de autonomía con $84 \%$ versus al escaso $27 \%$ alcanzado por Nursing Activity Scale ${ }^{14}$.

Por su parte, la autonomía en el trabajo se ha definido como "la libertad y discreción en la programación de las actividades laborales de la enfermera, lo cual incluye la habilidad de influir sobre el tiempo destinado al trabajo y descanso, designación de tareas, formulación y modificación de procedimientos, metas y modelos de evaluación” ${ }^{14}$. Dentro de este concepto se pueden clasificar los instrumentos Maastricht Autonomy Scale ${ }^{21}$; Control Scale $e^{22}$ Autonomy and Control Scale ${ }^{23}$, de los cuales se conocen valores de confiabilidad superiores a 0.87 (Tabla 1), de estas, sólo la escala Maastricht cuenta con reportes de su reproducibilidad ${ }^{14}$. En general se destaca que los contenidos de este tipo de instrumentos están fuertemente relacionados con los estándares de cuidado vigentes para el lugar y tiempo de su formulación, y obliga a reevaluar la pertinencia de sus ítems en la actualidad, de acuerdo a las características de las políticas locales del ejercicio de la profesión.

Por último, se mencionan las propuestas que buscaron responder a la diferenciación conceptual de la autonomía en subgrupos, es el caso del instrumento Autonomy:The Caring Perspective $(A C P)^{16}$ instrumento para la medición cuantitativa de las actitudes relacionadas con autonomía y comportamientos específicos de mujeres estudiantes de enfermería, basado en un modelo femenino; el cuál fue examinado en estudiantes de enfermería y otras profesiones, para luego ser validado por expertos. Al evaluar su reproducibilidad ${ }^{14}$, se presentaron altos valores del coeficiente de correlación de Pearson.

Existen otros conceptos como el Control Over Nursing Practice (CONP), el cual orienta su atención a las condiciones organizacionales para el desempeño de la labor enfermera y el concepto de independencia, que aunque se reconoce como un fenómeno diferente de la autonomía, algunos trabajos lo consideran una actividad (trabajar independientemente) que se realiza en el marco de la autonomía ${ }^{24}$; por lo anterior, es importante aclarar que la clasificación propuesta por las autoras de este trabajo, sólo incorpora instrumentos que han evaluado el constructo autonomía.

Finalmente, es importante destacar que todos los instrumentos han sido sometidos a procesos de validación a través de métodos cuantitativos y/o cualitativos; no obstante, aún existen cuestionamientos a los contenidos conceptuales de los mismos ${ }^{14}$, en este sentido es importante señalar que algunos estudios reportan medidas como es el coeficiente de correlación de Pearson para establecer la reproducibilidad de los instrumentos, el cual evalúa asociación entre variables cuantitativas y no permite identificar el acuerdo entre las medidas ${ }^{16}$; para este fin se recomiendan utilizar Kappa de Cohen (k) o Coeficiente de Correlación Intraclase (CCI) para variables nominales o continuas, respectivamente. En algunos estudios se encuentran reportes de coeficientes como el Alfa de Cronbach por subescalas, lo cual no brinda mayor información sobre la validez del constructo evaluado en cada instrumento ${ }^{18,17}$.

\section{Investigaciones sobre la Autonomía en Estudiantes de Enfermería}

A pesar de la importancia e interés del desarrollo de la autonomía en el profesional de enfermería desde la academia, pocas investigaciones han sido realizadas en estudiantes de enfermería. Los estudios encontrados se han realizado en diferentes grados académicos de Estados Unidos ${ }^{4,15}{ }^{24}$, Brasil ${ }^{25}$, ${ }^{26}$, Turquía ${ }^{5,27,28}$ y han sido comparados, principalmente, con estudiantes femeninos de otras carreras como medicina, odontología, educación, negocios, artes y ciencias ${ }^{4,5,15,28,}$ adicional a esto dos estudios incluyeron en su muestra profesores y monitores ${ }^{25,26}$. 
Todos ellos han realizado una única medición, la mayoría desarrollados desde un enfoque cuantitativo mediante un diseño de estudio de corte transversal ${ }^{4,5,15,27,29}$ y otros abordados desde la perspectiva cualitativa $^{10,30}$. En general, el objetivo principal de los estudios ha sido determinar el nivel de autonomía y en algunos casos comparar dicha autonomía con estudiantes de otras carreras ${ }^{5,27,28}$. Adicionalmente, algunas investigaciones también han explorado la correlación entre la autonomía y factores como: ocupación de los padres, promedio de calificaciones, prueba de aptitud académica, percepción de empoderamiento del liderazgo, percepción de autonomía, edad, masculinidad y comportamiento sumiso $^{15,28,29}$.

En cuanto al nivel de autonomía, un estudio realizado en 221 estudiantes de enfermería, obstetricia, medicina y odontología de último año, donde utilizaron el instrumento Sociotropy/Autonomy Scale (SAS) determinaron una media general de autonomía en estudiantes de enfermería de 77.33 \pm 15.19 puntos, esta es inferior a la autonomía de los estudiantes de obstetricia y medicina, pero superior al nivel de autonomía de los estudiantes de odontología ${ }^{5}$. Otro estudio realizado en 326 estudiantes de enfermería de diferentes niveles académicos, que emplearon la misma escala estableció una media general de autonomía de $83.03 \pm 14.51$ puntos. El nivel más alto de autonomía se encontró en los estudiantes de primer año (Media $84.87 \pm 14.44$ puntos), seguido de los de segundo año (Media 83.18 \pm 15.09 puntos), tercer año (Media 82.72 \pm 15.80 puntos) y cuarto año (Media 81.47 \pm 12.81 puntos ${ }^{27}$. Otro estudio realizado en 229 estudiantes de enfermería de diferentes niveles académicos determinó un promedio general de autonomía (SAS) de 66.16 \pm 19.46 y un promedio para el primer, segundo, tercer año y estudiantes senior de $71.06 \pm 15.47 ; 69.15 \pm 19.62 ; 66.09 \pm 20.70$; y $57.41 \pm 19.80$, respectivamente ${ }^{28}$.

Por otra parte, estudios que han explorado correlaciones con la autonomía como el de Boughn S, no encontró correlaciones significativas con la ocupación de los padres, promedio de calificaciones, prueba de aptitud académica, las escalas de feminidad y las puntuaciones de autonomía, sin embargo, si encontró una fuerte correlación entre las puntuaciones de la masculinidad y la autonomía ${ }^{15}$. Mailloux estableció un efecto directo significativo entre las percepciones de empoderamiento de liderazgo y la percepción de autonomía, al igual que la edad que tiene una relación directa significativa con la percepción de la autonomía ${ }^{29}$. Senturan L, et al. no encontró correlación significativa entre la autonomía y el comportamiento sumiso ${ }^{28}$ y Bronson S. no encontró asociación significativa entre el entorno de soporte de autonomía y la motivación autónoma, sin embargo, en un análisis de regresión la motivación autónoma y la inserción laboral fueron estadísticamente significativas4.

En relación a los estudios cualitativos se expone que la metodología de aprendizaje basado en problemas (ABP) promueve el aprendizaje continuo, el desarrollo de los estudiantes y la autonomía en el proceso de aprender a aprender ${ }^{25}{ }^{26}$. Adicionalmente, en esta metodología los profesores ayudan a los estudiantes a reconocer su estado inacabado ${ }^{25}$ y contribuir al desarrollo de relaciones dialógicas críticas y libres, con la oportunidad de reemplazar la imagen atemorizante del nuevo aprendizaje por algo que motive su curiosidad, lo que los lleva a ser sujetos autónomos ${ }^{31}$. La Tabla 2 resume metodologías y hallazgos de los estudios mencionados anteriormente.

\section{Conclusión}

La definición de autonomía profesional se ha realizado desde conceptos socialmente desarrollados, como lo son los roles de género (masculino y femenino), lo cual ha contribuido a una estigmatización de la profesión y confusión alrededor del constructo, puesto que, en el contexto profesional, la autonomía es una característica que trasciende los límites de la personalidad del individuo y es parte de los objetivos de formación.

En este sentido, otras propuestas han clasificado el concepto de autonomía según el nivel de educación, lo cual complejiza aún más su medición, puesto que suscita una diferenciación del mismo para cada formación académica y dista de aportar evidencia a la identificación de cambios en los niveles de 
Tabla 2. Investigaciones originales sobre autonomía en estudiantes de enfermería

\begin{tabular}{|c|c|c|c|c|}
\hline Autor-Año & País, & Diseño & Muestra & Principales Hallazgos \\
\hline $\begin{array}{l}\text { Boughn S. } \\
1988^{24}\end{array}$ & $\begin{array}{l}\text { Estados } \\
\text { Unidos }\end{array}$ & $\begin{array}{l}\text { Corte } \\
\text { transversal/ } \\
\text { Correlacional }\end{array}$ & $\begin{array}{l}1046 \text { estudiantes } \\
\text { mujeres de } \\
\text { programas } \\
\text { de pregrado } \\
\text { (enfermería, } \\
\text { educación, } \\
\text { negocios, artes } \\
\text { y ciencias) }\end{array}$ & $\begin{array}{l}\text { Estudiantes de enfermería presentaron puntajes más } \\
\text { bajos en autonomía y masculinidad, pero mayores en } \\
\text { feminidad que las de negocios, artes y ciencias. } \\
\text { Correlación entre las puntuaciones de la } \\
\text { masculinidad y la autonomía. }\end{array}$ \\
\hline $\begin{array}{l}\text { Boughn S. } \\
1992^{15}\end{array}$ & $\begin{array}{l}\text { Estados } \\
\text { Unidos }\end{array}$ & $\begin{array}{l}\text { Corte } \\
\text { transversal }\end{array}$ & $\begin{array}{l}219 \text { estudiantes } \\
\text { (enfermería, } \\
\text { educación, } \\
\text { negocios, } \\
\text { tecnología, artes } \\
\text { y ciencias) }\end{array}$ & $\begin{array}{l}\text { Estudiantes de enfermería y educación tenían } \\
\text { puntajes de feminidad más altos que los } \\
\text { estudiantes de tecnología, artes y ciencias. }\end{array}$ \\
\hline $\begin{array}{l}\text { Mailloux } \\
\text { CG. } 2006^{29}\end{array}$ & $\begin{array}{l}\text { Estados } \\
\text { Unidos }\end{array}$ & $\begin{array}{l}\text { Corte } \\
\text { transversal/ } \\
\text { Correlacional }\end{array}$ & $\begin{array}{l}198 \text { estudiantes } \\
\text { de enfermería } \\
\text { senior }\end{array}$ & $\begin{array}{l}\text { Diferencias estadísticamente significativas de edad en } \\
\text { los diferentes grupos de percepción de la autonomía }(\mathrm{t} \\
=2.652, \mathrm{p}=0.009) \text {, al igual que las percepciones de } \\
\text { empoderamiento de liderazgo }(\mathrm{t}=4.299, \mathrm{p}<0.001) \text {. }\end{array}$ \\
\hline $\begin{array}{l}\text { Karagozoglu } \\
\text { S. } 2008^{5}\end{array}$ & Turquía & $\begin{array}{l}\text { Corte } \\
\text { transversal }\end{array}$ & $\begin{array}{l}221 \text { Estudiantes } \\
\text { de enfermería, } \\
\text { obstetricia, } \\
\text { medicina y } \\
\text { odontología de } \\
\text { último año }\end{array}$ & $\begin{array}{l}\text { Media general de autonomía (Sociotropy/Autonomy } \\
\text { Scale-SAS) de todos los estudiantes fue de } 80.94 \\
\text { DE } \pm \text { 15.4. Los estudiantes de obstetricia tenían un } \\
\text { nivel más alto de autonomía (Media 83.48 DE } \pm \\
\text { 11.98), seguido de medicina (Media } 81.23 \mathrm{DE} \pm \\
\text { 15.36), enfermería (Media } 77.33 \mathrm{DE} \pm 15.19 \text { ). }\end{array}$ \\
\hline $\begin{array}{l}\text { Karagozoglu } \\
\text { S. } 2009^{27}\end{array}$ & Turquía & $\begin{array}{l}\text { Corte } \\
\text { transversal }\end{array}$ & 326 Estudiantes & $\begin{array}{l}\text { Media general de autonomía (SAS) fue de } 83.03 \mathrm{DE} \pm 14.51 \text {. } \\
\text { El nivel más alto de autonomía se encontró en los estudiantes } \\
\text { de primer año (Media } 84.87 \mathrm{DE} \pm 14.44 \text { ), seguido de los de } \\
\text { segundo año (Media 83.18 DE } \pm 15.09 \text { ), tercer año (Media } \\
82.72 \mathrm{DE} \pm 15.80 \text { ) y cuarto año (Media 81.47 DE } \pm 12.81 \text { ). }\end{array}$ \\
\hline $\begin{array}{l}\text { Da Silva AP, } \\
\text { et al. } 2010^{25}\end{array}$ & Brasil & $\begin{array}{l}\text { Cualitativa/ } \\
\text { Estudio } \\
\text { de caso }\end{array}$ & $\begin{array}{l}185 \text { Estudiantes } \\
\text { de enfermería, } \\
3 \text { docentes y } \\
2 \text { monitores }\end{array}$ & $\begin{array}{l}\text { El estudiante de enfermería necesita desarrollar su autonomía, } \\
\text { mediante el reconocimiento de su estado inacabado. }\end{array}$ \\
\hline $\begin{array}{l}\text { Senturan L, } \\
\text { et al. } 2012^{28}\end{array}$ & Turquía & $\begin{array}{l}\text { Corte } \\
\text { transversal }\end{array}$ & $\begin{array}{l}229 \text { Estudiantes } \\
\text { de enfermería } \\
\text { (primer, } \\
\text { segundo, tercer } \\
\text { año y estudiantes } \\
\text { senior). }\end{array}$ & $\begin{array}{l}\text { Media general de autonomía (SAS) de los estudiantes } \\
\text { fue } 66.16 \mathrm{DE} \pm 19.46 \text {. El promedio de puntaje en } \\
\text { la escala de comportamiento sumiso (Submissive } \\
\text { Behaviour Scale) fue } 32.87 \mathrm{DE} \pm 7.54 \text {. Diferencia } \\
\text { estadísticamente significativa en el promedio total } \\
\text { de puntuaciones para autonomía según la clase. }\end{array}$ \\
\hline $\begin{array}{l}\text { Waterkemper } \\
\text { R, et al. } \\
2014^{31}\end{array}$ & Brasil & $\begin{array}{l}\text { Cualitativa / } \\
\text { basado en el } \\
\text { modelo de } \\
\text { Ludke-André }\end{array}$ & $\begin{array}{l}14 \text { Estudiantes } \\
\text { de enfermería } \\
\text { de la asignatura } \\
\text { Fundamentos } \\
\text { para el Cuidado } \\
\text { Profesional }\end{array}$ & $\begin{array}{l}\text { Los estudiantes de enfermería, en la medida en que se } \\
\text { sienten libres, tienen la oportunidad de reemplazar la imagen } \\
\text { atemorizante del nuevo aprendizaje por algo que motive } \\
\text { su curiosidad, lo que los lleva a ser sujetos autónomos. }\end{array}$ \\
\hline $\begin{array}{l}\text { Campos LRG, } \\
\text { et al. } 2014^{26}\end{array}$ & Brasil & $\begin{array}{l}\text { Cualitativo/ } \\
\text { Método } \\
\text { Bricolage }\end{array}$ & $\begin{array}{l}16 \text { Estudiantes } \\
\text { de enfermería } \\
12 \text { Tutores }\end{array}$ & $\begin{array}{l}\text { El Aprendizaje Basado en Problemas (ABP) favorece la } \\
\text { construcción de conocimientos mediante el uso de saberes } \\
\text { y experiencias que se comparten en grupos pequeños, } \\
\text { a través del proceso de teorización, y por medio de los } \\
\text { conocimientos relevantes que se puedan aplicar a la práctica. }\end{array}$ \\
\hline $\begin{array}{l}\text { Bronson } \\
\text { S. } 2016^{4}\end{array}$ & $\begin{array}{l}\text { Estados } \\
\text { Unidos }\end{array}$ & $\begin{array}{l}\text { Corte } \\
\text { transversal/ } \\
\text { Correlacional }\end{array}$ & $\begin{array}{l}150 \text { Estudiantes } \\
\text { de enfermería } \\
\text { de último año } \\
\text { ( } 4 \text { universidades } \\
\text { de la Florida) }\end{array}$ & $\begin{array}{l}\text { El entorno de aprendizaje sólo fue de apoyo moderado } \\
\text { en la motivación de los estudiantes (Media } 70.60 \mathrm{DE} \pm \\
18.99 \text { ). No se encontraron asociaciones significativas } \\
\text { entre el entorno de soporte de autonomía y la motivación } \\
\text { autónoma }(\mathrm{r}=0.034, \mathrm{p}=0.676) \text {. Correlaciones y análisis } \\
\text { de regresión de la motivación autónoma y la inserción } \\
\text { laboral fueron significativas (F }(2,147)=28.28, \mathrm{p}= \\
\text { 0.000). La comparación de los grupos de participantes de } \\
\text { cada universidad reveló entornos de aprendizaje de apoyo. }\end{array}$ \\
\hline
\end{tabular}


un único constructo, bajo la influencia de variables tan importantes como la educación; sin embargo, con el estado actual del conocimiento no se cuenta con un instrumento de medición sensible a estos cambios.

Además, de acuerdo a lo que se encontró como resultados de investigaciones sobre autonomía en estudiantes de enfermería, también se evidenció la necesidad de: 1) desarrollar estudios que validen un instrumento para determinar la autonomía profesional en población hispanohablante; 2) estudios de diseños longitudinales prospectivos (tipo cohorte) que permitan evaluar el comportamiento de la autonomía en los mismos estudiantes a través del avance en los diferentes niveles académicos, este diseño también favorecería la confirmación de hallazgos previos derivados de estudios correlacionales, que han sugerido que a medida que aumenta el nivel de conocimiento, el nivel educativo, y la auto-confianza de una persona, se fortalece el pensamiento crítico, la resolución de problemas y la toma de decisiones independientes; 3 ) estudios rigurosos que permitan establecer los factores asociados a un mayor nivel de autonomía y finalmente, 4) estudios que diseñen y evalúen intervenciones o metodologías que contribuyan a desarrollar la autonomía en los estudiantes de enfermería.

\section{Responsabilidades éticas}

Protección de personas y animales. Para la elaboración de este artículo se trabajó con documentos no con personas.

Financiamiento

Ninguno.

Conflicto de intereses

Los autores declaran no tener conflicto de intereses.

\section{Referencias}

1. Consejo Internacional de Enfermeras. La definición de enfermería. Ginebra: CIE; 2017 [consultado 19 marzo 2018]. Disponible en: https://bit.ly/1FeyuQ1

2. Lopera-de Peña Á. 10 Años De La Ley 911 De 2004. Av.Enferm. 2014; 32(2): 1-11.

3. Martínez CL, Paravic T. Autonomía Profesional: Factor clave para el ejercicio de la enfermería basada en la evidencia. Index enferm. 2016; 25(1): 42-6.

4. Bronson S. Autonomy support environment and autonomous motivation on nursing student academic performance: An exploratory analysis. Nurse Educ Today. 2016; 44: 103-8. https://doi.org/10.1016/j.nedt.2016.05.013

5. Karagozoglu S. Level of autonomy of Turkish students in the final year of university baccalaureate degree in health related fields. Nurs Outlook. 2008; 56(2): 70-7. https://doi.org/10.1016/j.outlook.2007.11.002

6. Blanchfield KC, Biordi DL. Power in practice: a study of nursing authority and autonomy. Nurs Adm Q. 1996; 20(3): 42-9.

7. Tapp D, Stansfield K, Stewart J. La autonomía en la práctica de enfermería. Aquichán. 2005; 5(1): 114-27.

8. Ballou KA. A concept analysis of autonomy. J Prof Nurs. 1998; 14(2): 102-10.

9. Mazo Álvarez HM. La autonomía: principio ético contemporáneo. rev.colomb.cienc.soc. 2012; 3(1): 115-32. https://doi.org/10.21501/issn.2216-1201

10. Pankratz L, Pankratz D. Nursing autonomy and patients' rights: Development of a nursing attitude scale. J Health Soc Behav. 1974; 15(3): 211-6.

11. Wade GH. A model of the attitudinal component of professional nurse autonomy. J Nurs Educ. 2004; 43(3): 116-24. 
12. Ward DJ, Schaal M, Sullivan J, et al. The Jefferson Scale of Attitudes toward Physician-Nurse Collaboration: A study with undergraduate nursing students. J Interprof Care. 2008; 22(4): 375-86. https://doi.org/10.1080/13561820802190533

13. Hernández-Martín F, Gallego-Lastra R, Alcaraz- Gonzalez S, et al. La enfermería en la historia. Un análisis desde la perspectiva profesional. Cul cuid. 1997; 2(1): 21-35.

14. Weston MJ.Validity of Instruments for Measuring Autonomy and Control Over Nursing Practice. J Nurs Scholarsh. 2009; 41(1): 87-94. https://doi.org/10.1111/j.1547-5069.2009.01255.x

15. Boughn S. Nursing students rank high in autonomy at the exit level. J Nurs Educ. 1992; 31(2): 58-64.

16. Boughn S. An Instrument for Measuring Autonomy- Related Attitudes and Behaviors in Women Nursing Students. J Nurs Educ. 1995; 34(3): 106-13.

17. Cassidy VA, Oddi LF. Professional Autonomy and Ethical Decision Making Among Graduate and Undergraduate Nursing Majors. J Nurs Educ. 1988; $27(9)$ : 405-10.

18. Cassidy VR, Oddi LF. Professional autonomy and ethical decision making among graduate and undergraduate nursing majors: A Replication. J Nurs Educ. 1991; 30(4): 149-51.

19. Schutzenhofer K. Measuring professional autonomy in nurses. In: Strickland OL, Dilorio C. Measurement of Nursing Outcomes. New York: Springer; 1988. P. 3-18.

20. Papathanassoglou E, Tseroni M, Karydaki A, et al. Practice and clinical decision-making autonomy among Hellenic critical care nurses. J Nurs Manag. 2005; 13(2): 154-64.

https://doi.org/10.1111/j.1365-2934.2004.00510.x

21. De Jonge J, Bosma $\mathrm{H}$, Peter R, et al. Job strain, effort-reward imbalance and employee well-being: a large-scale cross-sectional study. Soc sci med. 2000; 50(9): 1317-27.

22. Dwyer D, Ganster D. The effects of job demands and control on employee attendance and satisfaction. J Organ Behav. 1991; 12(7): 595-8. https://doi.org/10.1002/job.4030120704

23. Haynes $\mathrm{C}$, Wall T, Bolden $\mathrm{R}$, et al. Measures of perceived work characteristics for health services research: Test of a measurement model and normative data. Br J Health Psychol. 1999; (4): 257-75. https://doi.org/10.1348/135910799168614

24. Boughn S. A lack of autonomy in the contemporary nursing student: a comparative study. J Nurs Educ. 1988; 27(4): 150-5. https://doi.org/10.3928/0148-4834-19880401-04

25. Schell Da Silva APS, Pedro ENR. Autonomy in nursing students process of knowledge construction: the educational chat as a teaching tool. Rev. Lat.-Am. Enferm. 2010; 18(2): 210-6.

https://doi.org/10.1590/S0104-11692010000200011

26. Campos LRG, Ribeiro MRR, Depes VBS. Autonomia do graduando em enfermagem na (re)construção do conhecimento mediado pela aprendizagem baseada em problemas. Rev Bras Enferm. 2014; 67 (5): 818-24. https://doi.org/10.1590/0034-7167.2014670521

27. Karagözoğlu Ş. Nursing students' level of autonomy: A study from Turkey. Nurse Educ Today. 2009; 29(2): 176-87. https://doi.org/10.1016/j.nedt.2008.08.002

28. Senturan L, Kose S, Sabuncu N, et al. Autonomy and submissive behaviour among students at the college of nursing. HealthMED. 2012; 6(8): 2741-8.

29. Mailloux CG. The extent to which students' perceptions of faculties' teaching strategies, students' context, and perceptions of learner empowerment predict perceptions of autonomy in BSN students. Nurse Educ Today. 2006; 26(7): 578-85. https://doi.org/10.1016/j.nedt.2006.01.013

30. De-Luca C. Implicaciones de la Formación en la Autonomía del Estudiante Universitario. Rev Electron Investig Psicoeduc Psigopedag 2009; 7(18): 901-22.

31. Waterkemper R, Lenise do Prado M, Medina-Moya JL. From "being to the self" to "become to be": on development of autonomy construction in nursing students. Invest educ enferm. 2014; 32(1): 33-40. https://doi.org/10.1590/S0120-53072014000100004 